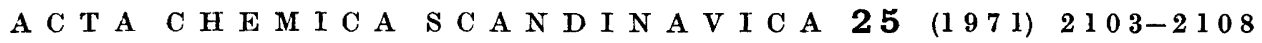

\title{
Comparative Structural Studies on the M-Antigen from Salmonella typhimurium, Escherichia coli and Aerobacter cloacae
}

\author{
PER J. GAREGG, ${ }^{a}$ B ENGT LINDBERG, ${ }^{\text {a }}$ THORSTEN ONNa and \\ I. W. S U T H E R L A N D ${ }^{b}$
}

\begin{abstract}
a Institutionen för organisk kemi, Stockholms Universitet, S-11327 Stockholm, Sweden, and ${ }^{\mathrm{b}}$ Department of General Microbiology, University of Edinburgh, Edinburgh, Great Britain
\end{abstract}

\begin{abstract}
Structural studies have been carried out on the M-antigen isolated from $S$. typhimurium 395MRO (M1 and M2), $E$. coli K12 (S53 and S61), two other $E$. coli strains (S15 and S20), and $A$. cloacae NCTC 035, 259 and 923. They all seem to have the same basic structure and are composed of hexasaccharide repeating units. All but one (S. typhimurium 395MRO-M1) contain $O$-acetyl groups. Structural variation is provided by various alkylidene groups, linked to the terminal D-galactopyranose residues. These are ethylidene, carboxyethylidene, and methylene groups. The former are linked to positions 3 and 4 of the D-galactose residue, while carboxyethylidene groups can alternatively be linked to positions 3,4 or 4,6 . The methylene groups are linked to positions 4 and 6 .
\end{abstract}

A $\mathrm{n}$ acidic polysaccharide, M-antigen or colanic acid, is produced by several Enterobacteriaceae, when these are grown under appropriate conditions. The early work on the structure of these polysaccharides has beensum marized. ${ }^{1}$ In recent investigations, the M-antigen from $E$. coli $\mathrm{K} 12(\mathrm{~S} 53)^{2,3}$ and S. typhimurium 395MRO (M1 and M2) (,5 $^{4}$ have been studied by fragmentation

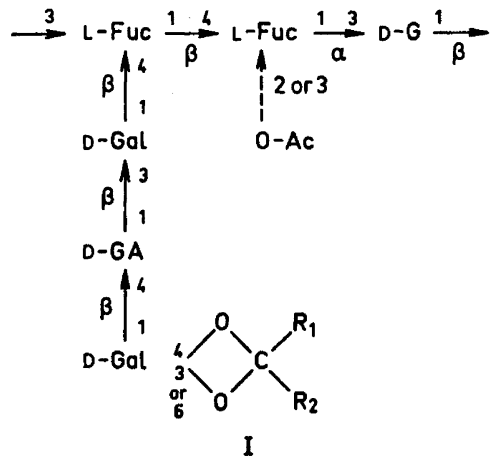

Acta Chem. Scand. 25 (1971) No. 6 
analysis, methylation analysis, and other methods. These studies demonstrated that the different $M$-antigens have a similar structure and are composed of hexasaccharide repeating units. A detailed structure (I) for this hexasaccharide repeating unit could be proposed.

The terminal $\mathrm{D}$-galactopyranose residue in $\mathrm{I}$ was substituted in different manners in the M-antigens investigated. In the $E$. coli $\mathrm{K} 12$ (S53) polysaccharide it carried a 4,6-O-carboxyethylidene group, ${ }^{2,3}$ in the $S$. typhimurium 395MROM1 polysaccharide a 3,4-O-ethylidene group, ${ }^{4,5}$ and in the $S$. typhimurium 395MRO-M2 a 3,4-carboxyethylidene group. 5 Furthermore, $O$-acetyl groups were present in all polysaccharides but one, namely that from $S$. typhimurium 395MRO-M1. In order to examine more thoroughly the general validity of structure $I$ and possible variations in the nature and mode of linkage of the $O$-alkylidene groups, an exploratory screening of the M-antigens isolated from various enterobacteria has been carried out.

\section{RESULTS AND DISCUSSION}

M-Antigens were prepared from Salmonella typhimurium 395MRO, M1 and M2, Escherichia coli K12 (S53 and S61), S15 and S20, and Aerobacter cloacae NCTC 035, 259 and 923, and subjected to sugar analysis (Table 1).

Table 1. Sugar composition of the polysaccharides. Figures for all monosaccharides represent mol\% of detected sugars.

M-antigen from

S. typhimurium 395MRO-M1

S. typhimurium 395MRO-M2

E. coli (S15)

E. coli (S20)

E. coli K12 (S53)

E. coli K12 (S61)

A. cloacae NCTC 035

$A$. cloacae NCTC 259

A. cloacae NCTC 923

Fuc
$\mathbf{4 3}$
$\mathbf{4 2}$
$\mathbf{4 0}$
$\mathbf{3 8}$
$\mathbf{3 9}$
$\mathbf{4 1}$
$\mathbf{3 8}$
$\mathbf{3 7}$
$\mathbf{3 6}$

$\begin{array}{cc}\text { Gal } & \text { G } \\ \mathbf{3 0} & \mathbf{2 7} \\ \mathbf{3 3} & \mathbf{2 5} \\ \mathbf{3 2} & \mathbf{2 8} \\ \mathbf{3 2} & \mathbf{3 0} \\ \mathbf{2 9} & \mathbf{3 2} \\ \mathbf{2 9} & \mathbf{3 0} \\ \mathbf{3 6} & \mathbf{2 6} \\ \mathbf{3 8} & \mathbf{2 5} \\ \mathbf{3 2} & \mathbf{3 2}\end{array}$

D-Glucuronic acid and a varying part of the D-galactose, probably present as an aldobiouronic acid, are not accounted for in these analyses. Nevertheless, the results demonstrate that the polysaccharides are closely related. Studies on the M-antigens from several other bacteria were attempted. The M-antigens, however, often have a low solubility in water, and are difficult to purify. Only studies on the M-antigens which, according to their sugar composition, were reasonably pure, are included in the present communication. O-Acetyl groups were present in all M-antigens except in that from $S$. typhimurium 395MRO-Ml, as indicated by IR absorption at $1720 \mathrm{~cm}^{-1}$ and confirmed by the demonstration of methyl acetate in the product of methanolysis by GLC.

The nature of the carbonyl compounds (arising from alkylidene groups) (Table 2), released from the polysaccharides on mild acid hydrolysis, was determined by performing this hydrolysis in the presence of 2,4-dinitrophenyl- 
STUDIES ON M-ANTIGEN

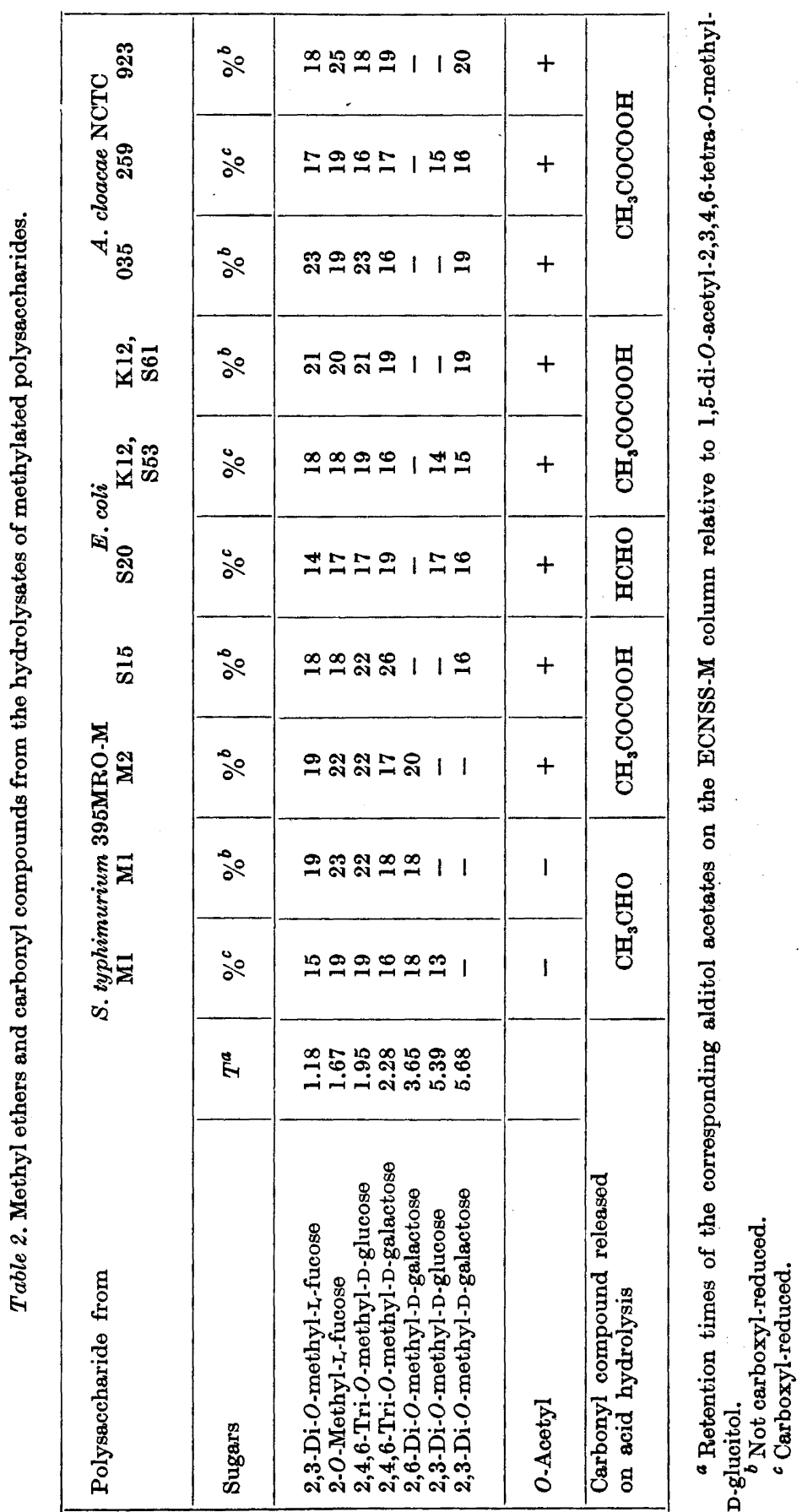

Acta Chem. Scand. 25 (1971) No. 6 
hydrazine. The identities of the hydrazones found were established by TLC, GLC, PC and by MS.

The M-antigens were methylated by treatment with methylsulphinyl sodium and methyl iodide in methyl sulphoxide, ${ }^{6}$ the fully methylated polysaccharide hydrolyzed to a mixture of methylated sugars, and these analyzed, as their alditol acetates, by GLC ${ }^{7}-\mathrm{MS}^{8}$ (Table 2). Representative chromatograms are given in Figs. 1 and 2. Some of the methylated polysaccharides

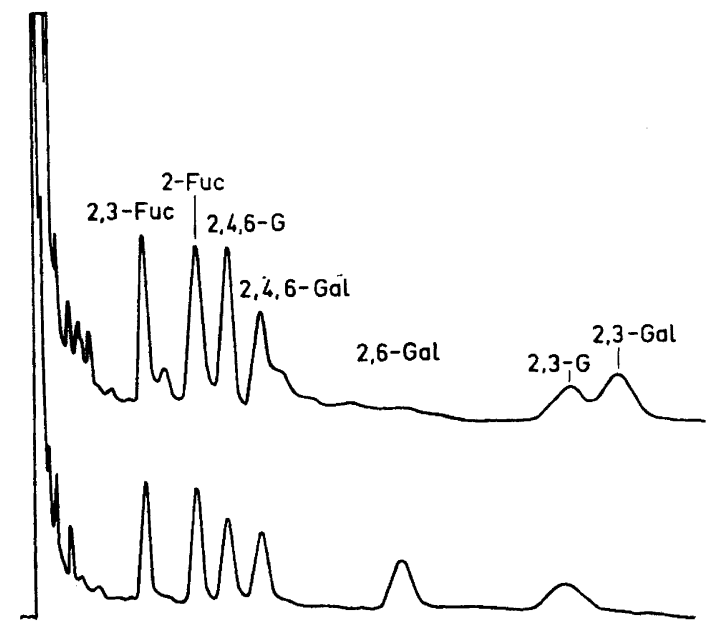

Fig. 1. GLC-separation of methylated sugars, as their alditol acetates, obtained from the hydrolysates of the fully methylated, carboxyl-reduced polysaccharides from $E$. coli K12 (S53), upper, and from $S$. typhimurium 395MRO-MI, lower. 2,3-Fuc means the alditol derived from 2,3-di- $O$-methyl-L-fucose, etc. Peaks not accounted for are of noncarbohydrate nature (demonstrated by mass spectrometry).

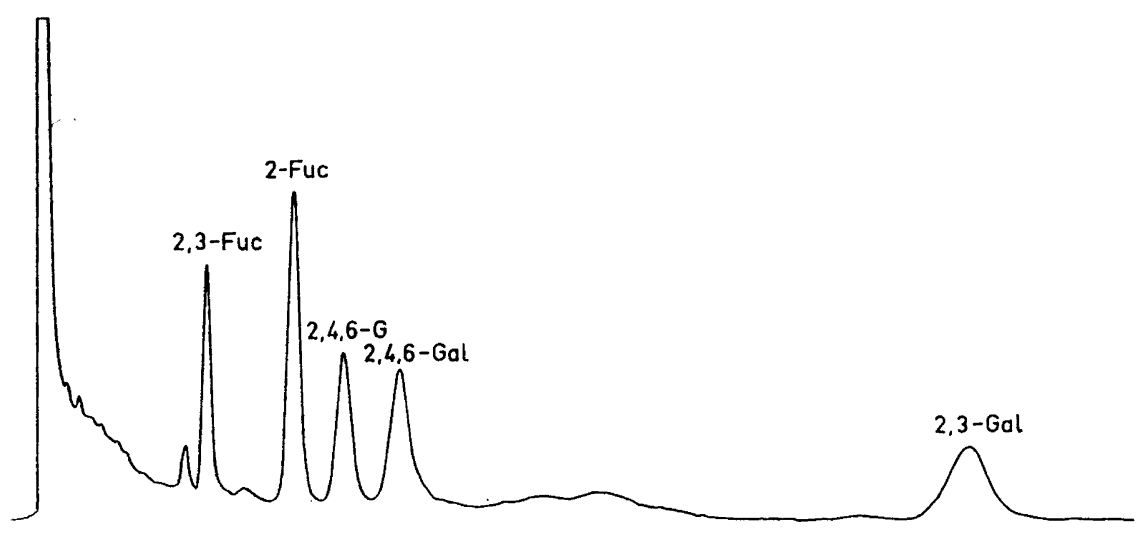

Fig. 2. GLC-separation of methylated sugars, as their alditol acetates, obtained from the hydrolysate of the fully methylated, not carboxyl-reduced polysaccharide from $A$. cloacae NCTC 923. 2,3-Fuc means the alditol derived from 2,3-di-O-methyl-1,-fucose, etc. Peaks not accounted for are of non-carbohydrate nature (demonstrated by mass spectrometry). 
were subjected to carboxyl reduction before hydrolysis. In these, but not in the others, the D-glucuronic acid residues are accounted for (as 2,3-di- $O$ methyl-D-glucose).

The various methylation analyses reveal no significant deviation from structure I. There is, however, considerable variation in the nature of the substitution of the terminal $\mathrm{D}$-galactose residues, as revealed by the isolation of different carbonyl compounds on acid hydrolyses and by the presence of either 2,3-di- $O$-methyl-D-galactose (4,6-residues) or 2,6-di- $O$-methyl-D-galactose $(3,4$-residues) in the methylation analysis. Six of the nine polysaccharides contain a 4,6-O-carboxyethylidene group (II).
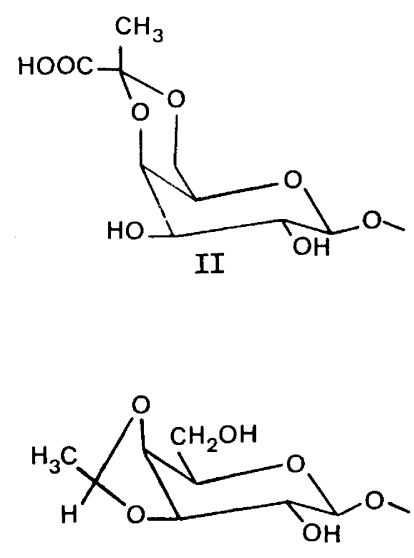

IV
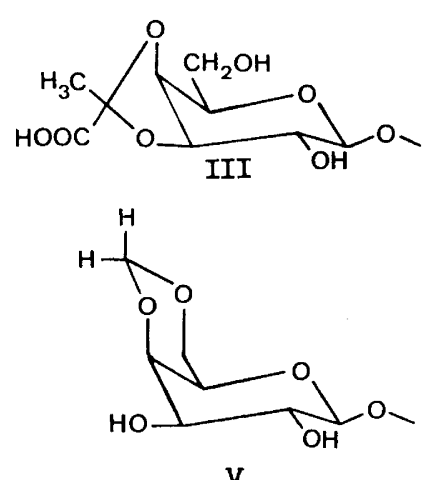

Y

In one, the S. typhimurium 395MRO-M2, the carboxyethylidene group is linked to the 3 and 4 positions (III), ${ }^{5}$ and in another, S.typhimurium 395MRO$\mathrm{M} 1$, an ethylidene group is linked to the same positions (IV). ${ }^{4}$ Finally, in the $E$. coli (S20) M-antigen, a methylene group is linked to positions 4 and $6(\mathrm{~V})$.

The partial structure II has been observed in other polysaccharides. ${ }^{9}$ Pyruvic acid, linked to the 3 and 4 positions of a D-galactose residue (III) has not been found in other polysaccharides. Acetals of acetaldehyde (IV) and formaldehyde (V) have, to the best of our knowledge, not previously been demonstrated in polysaccharides or in other naturally occurring carbohydrates. A methylene derivative of an inositol ${ }^{10}$ is, however, known and also methylene derivatives of 1,2-dihydroxybenzene derivatives.

By acetalisation (IV) or ketalisation (II, III), a new asymmetric carbon atom is introduced. The configuration at the ketalic carbon atom of the 4,6- $O$-carboxyethylidene- $D$-galactose residue in the polysaccharide from Corynebacterium insidiosum ${ }^{11}$ has been determined. It does not seem unreasonable to assume that the corresponding units (II) present in some of the M-antigens have the same configuration. Nothing is, however, known about the configuration at the ketalic or acetalic carbon atom in III and IV, present in the other M-antigens.

Acta Chem. Scand. 25 (1971) No. 6 
Attempts to determine the positions of the $O$-acetyl groups, using the method devised by de Belder and Norrman, ${ }^{12}$ were unsuccessful, as the polysaccharides did not dissolve on treatment with methyl vinyl ether and $p$ toluenesulphonic acid in methyl sulphoxide. The results of the periodate oxidation of one of them (S. typhimurium 395MRO-M2) indicate that the non-branched fucose residue carries an $O$-acetyl group. ${ }^{5}$

Added in proof. A methylation analysis, as described above, of Salmonella typhimurium \$4. 1543 gave the following result (not carboxyl reduced): 2,3-di-O-methylfucose : 2. $O$. methylfucose : 2,4,6-tri-O-methyglucose : 2,4,6-tri-O-methylgalactose : 2,6-di-O. methylgalactose $20: 19: 21: 21: 19$. Mild acid hydrolysis released pyruvic acid. This polysaccharide thus is similar to that from Salmonella typhimurium 395 MRO-M2. It differs in the carboxyethylidene residues from the polysaccharides of $E$. coli $\mathrm{K} 12$ and $A$. cloaceae NCTL 5920 to which earlier work ${ }^{2,3}$ had shown similarities in carbohydrate structure.

\section{EXPERIMENTAL}

Concentrations were carried out in a vacuum at bath temperatures below $40^{\circ}$.

The formaldehyde, derived from the methylene acetal in $E$. coli (S20), was determined as its 2,4-dinitrophenylhydrazone by methods described in a previous paper. The m.p. of the crystals was $160-163^{\circ}$. Authentic formaldehyde 2,4-dinitrophenylhydrazone melts at $166^{\circ}$.

All the other various methods used are identical with those described in a previous paper, ${ }^{5}$ and their description will therefore not be repeated here.

Acknowledgements. This work was supported by grants from the Swedish Medical Research Contncil (No. B69-13X-2522-01), the Swedish Natural Science Research Council, Harald Jeanssons Stiftelse, and from Sigurd och Elsa Goljes minne. We are indebted to Dr. D. A. Rees for valuable criticism of the manuscript.

\section{REFERENCES}

1. Lüderitz, O., Jann, K. and Wheat, R. Compreh. Biochem. A 26 (1968) 208.

2. Sutherland, I. W. Biochem. J. 115 (1969) 935.

3. Lawson, C. J., McCloary, C. W., Nakada, H. J., Rees, D. A., Sutherland, I. W. and Wilkinson, J. F. Biochem. J. 115 (1969) 947.

4. Garegg, P. J., Lindberg, B., Onn, T. and Holme, T. Acta Chem. Scand. 23 (1969) 2194.

5. Garegg, P. J., Lindberg, B., Onn, T. and Holme, T. Acta Chem. Scand. 25 (1971) 1185.

6. Hekomori, S. J. Biochem. (Tokyo) 55 (1964) 205.

7. Björndal, H., Lindberg, B. and Svensson, S. Acta Chem. Scand. 21 (1967) 1801.

8. Björndal, H., Lindberg, B. and Svensson, S. Carbohyd. Res. 5 (1967) 433.

9. Gorin, P. A. J. and Spencer, J. T. Can. J. Chem. 42 (1964) 1230.

10. Namiki, M., Isono, K. and Suzuki, S. J. Antibiotics (Japan) Ser. A 10 (1957) 160.

11. Gorin, P. A. J. and Ishikawa, T. Can. J. Chem. 45 (1967) 521.

12. de Belder, A. N. and Norrman, B. Carbohyd. Res. 8 (1968) 1.

Received November 3, 1970. 